\title{
Estudo longitudinal da relação entre o crescimento mandibular e o crescimento estatural em indivíduos com Classe II esquelética
}

\author{
Guilherme THIESEN*, Marcus Vinicius Neiva Nunes do REGO*, Eduardo Martinelli Santayana de LIMA**
}

\begin{abstract}
Resumo
O crescimento corporal de crianças e adolescentes e sua relação com a aceleração do crescimento do complexo craniofacial constituem assunto de enorme interesse para o ortodontista, uma vez que a instituição de uma terapia que vise controlar o desenvolvimento maxilo-mandibular é baseada no estudo da idade esquelética do paciente e sua conseqüente maturação somática. Este estudo objetivou assim avaliar a correlação existente entre o crescimento estatural e o crescimento mandibular em 30 indivíduos com Classe II esquelética, não tratados ortodonticamente, acompanhados longitudinalmente junto ao Burlington Growth Study - Canadá. As documentações seriadas foram obtidas aos 6, 9, 12, 14 e 16 anos de idade cronológica, constando de telerradiografias de perfil e fichas clínicas com dados cadastrais. Os resultados indicaram que o pico de crescimento estatural, bem como o maior incremento médio da maioria das medidas mandibulares avaliadas, ocorreu para o gênero feminino, no período entre 9 e 12 anos, e para o gênero masculino, entre 12 e 14 anos. Além disso, foi verificado que, dentre todas as medidas analisadas, a velocidade média de incremento no comprimento da mandíbula demonstrou a correlação mais consistente com a velocidade média de crescimento em altura destes indivíduos, principalmente para o gênero masculino durante seu pico de crescimento $(r=0,758)$.
\end{abstract}

Palavras-chave: Classe II. Crescimento mandibular. Crescimento estratural. Surto de crescimento facial.

\section{INTRODUÇÃO E REVISÃO DE LITERATURA}

A compreensão dos eventos relacionados ao desenvolvimento físico dos indivíduos é de suma importância na Ortodontia clínica. Deste modo, o crescimento corporal do adolescente e sua relação com a aceleração do crescimento do complexo craniofacial constituem assunto de enorme inte- resse para o ortodontista, uma vez que a instituição de uma meta terapêutica que vise controlar o desenvolvimento maxilo-mandibular é baseada no estudo da idade esquelética do paciente e sua conseqüente maturação somática.

O peso, a estatura, as idades óssea e dentária, têm sido considerados na avaliação do crescimento e

\footnotetext{
* Mestres em Ortodontia e Ortopedia Facial pela Pontifícia Universidade Católica do Rio Grande do Sul - PUCRS.

** Mestre e Doutor em Ortodontia pela UFRJ, Professor Coordenador do Curso de Mestrado em Ortodontia e Ortopedia Facial da Pontifícia Universidade Católica do Rio Grande do Sul - PUCRS.
} 
desenvolvimento craniofacial1,2,3,4,18,27,30. Algumas dessas variáveis mostram-se ineficientes, pois não apresentam íntima relação com a maturação facial, como por exemplo, as idades dentária e cronológica ${ }^{10,32}$.

Björk $^{8,9,10}$ verificou que a curva de velocidade de crescimento estatural de um indivíduo é um instrumento adequado para avaliar o crescimento facial. A correlação entre os surtos de crescimento estatural e craniofacial indica que a taxa de crescimento estatural pode ser utilizada para determinar a época de aceleração de crescimento, tanto estatural quanto craniofacial. Outros estudos ratificaram esta estreita relação entre o crescimento corporal e o craniofacial, estabelecendo ainda que dentre todas as dimensões faciais, aquela que apresentou maior correlação com a altura corporal foi o comprimento ântero-posterior da mandíbula ${ }^{12,18,35}$.

Tanner ${ }^{34}$ esclarece que o surto de crescimento puberal é um fenômeno constante a todos os indivíduos, variando, entretanto, na intensidade, duração e idade cronológica de ocorrência. Nos indivíduos do gênero masculino, ocorre em média entre 12 anos e seis meses e 15 anos. No gênero feminino, o surto de crescimento puberal acontece, em média, entre os 10 anos e 6 meses e 13 anos, sendo menor em magnitude e intensidade que para o gênero masculino. Além disso, este tende a iniciar-se, em média, cerca de 2 anos antes do início do surto de crescimento puberal masculino, fato este também evidenciado por Hioki ${ }^{16} \mathrm{ePike}^{26}$.

Ao avaliar longitudinalmente uma série de medidas faciais, Nanda ${ }^{24}$ formulou gráficos e curvas de crescimento médio de acordo com seus achados. $\mathrm{O}$ referido autor registrou aumento generalizado na velocidade de crescimento craniofacial durante o surto de crescimento puberal, embora o período tanto do início quanto do pico deste crescimento fossem diferentes para as várias dimensões e medidas de um mesmo paciente. A aceleração máxima circumpuberal do crescimento facial ocorreu aproximadamente nove meses após aquela em altura corporal.

Os achados de Bambha ${ }^{3}$, em geral, apresentaram concordância com aqueles previamente estabeleci- dos por $\mathrm{Nanda}^{24}$, e o autor ainda sugeriu que seria possivel estimar-se o tempo exato do início do surto de crescimento facial puberal através da observação do período de início da aceleração de crescimento em altura, que ocorreria alguns meses antes.

Hunter ${ }^{18}$, por sua vez, realizou um estudo que veio a contrariar os resultados encontrados por $\mathrm{Nanda}^{24} \mathrm{e}$ Bambha $^{3}$. O autor concluiu que o crescimento facial máximo era coincidente com o crescimento máximo em altura, para a maioria dos indivíduos do seu estudo. O comprimento ântero-posterior da mandíbula, dentre todas as medidas faciais estudadas, demonstrou a correlação mais consistente com o crescimento em altura, fato este também observado por diversos outros autores ${ }^{29,32,35}$. Daigle ${ }^{12}$ observou que, no gênero masculino, em $86 \%$ dos casos, o pico de crescimento mandibular ocorreu ao mesmo tempo ou logo após o pico de crescimento em altura, obtendo assim o mesmo resultado encontrado por Hunter ${ }^{18}$ para ambos os gêneros.

As abordagens terapêuticas que visem a correção das desarmonias craniofaciais devem, portanto, levar em consideração o estágio de desenvolvimento esqueletal que o paciente apresenta, pois, os resultados estarão intimamente relacionados a esta circunstância ${ }^{18,31}$. Na prática ortodôntica, um confiável meio para determinar o desenvolvimento físico dos indivíduos é a avaliação da idade esquelética. Vários autores já observaram a estreita relação existente entre a idade em que ocorre a maior taxa de crescimento corporal em altura e o período de ossificação dos ossos do corpo ${ }^{1,2,10,14,18}$. Assim, a forte correlação entre o pico de crescimento estatural e certos estágios de ossificação na mão e no punho já se encontra bem estabelecida na literatura ${ }^{38}$.

Griwalsky ${ }^{15}$ encontrou correlações significativas entre a maturidade estatural e o comprimento mandibular em meninas canadenses dos 6 aos 12 anos. Contudo, no período seguinte, entre 12 e 20 anos, não houve correlação significativa. A associação entre as maturidades em estatura e mandibular foi $70 \%$ maior aos 12 anos do que aos 20 anos. Thompson e Popovich ${ }^{35}$ relataram coeficiente de 
correlação máximo de 0,62 aos 11 anos de idade. Gradualmente, esse valor foi diminuindo até 0,36 aos 18 anos.

Reed $^{29}$ correlacionou os incrementos ocorridos na altura corporal e no comprimento mandibular, entre 9 e 16 anos, e encontrou coeficiente de correlação de 0,68 para os indivíduos do gênero masculino e de 0,60 para os do gênero feminino. Nos períodos parciais, os valores maiores surgiram entre 14 e 16 anos, atingindo 0,82 e 0,80 nos gêneros masculino e feminino, respectivamente. Bishara et al. ${ }^{4}$ encontraram valor máximo de correlação entre essas medidas de 0,83. Anderson, Thompson e Popovich ${ }^{1}$ observaram que a maior correlação foi verificada justamente no período de pico de crescimento, tanto para a estatura como para o comprimento mandibular.

A implicação destes e outros estudos é que os fatores responsáveis pelo crescimento e maturação corporal também controlam o crescimento e desenvolvimento da face do indivíduo.

Neste contexto, o crescimento craniofacial nas más oclusões de Classe II constitui um assunto de grande interesse, visto que esse tipo de alteração morfológica na relação entre as bases apicais constitui uma significante porcentagem dos casos na prática ortodôntica ${ }^{5}$.

Ao avaliar uma amostra de indivíduos portadores de má oclusão de Classe II, no estágio de dentadura mista, McNamara ${ }^{21}$ descreveu as seguintes características morfológicas tridimensionais: discrepância maxilar transversa, retrusão esquelética mandibular e altura facial anterior inferior aumentada. De acordo com Dale ${ }^{13}$, a arquitetura crânio-facial dos indivíduos portadores de Classe II esquelética pode ser composta por retrognatismo mandibular, prognatismo maxilar ou pela combinação das duas possibilidades. Desta forma, o estudo do crescimento mandibular torna-se extremamente importante, visto que muitas vezes o ortodontista conta com este fator para a correção deste tipo de má oclusão.

Segundo Lima ${ }^{20}$, o estudo do crescimento de indivíduos portadores de Classe II esquelética, não tratados ortodonticamente, é fundamental para a avaliação das alterações induzidas pela mecanoterapia, visto que o crescimento desses indivíduos é extremamente variável, de difícil predição e diferente quando comparado ao crescimento de indivíduos com oclusão normal, o que poderia dificultar a interpretação do real efeito da mecanoterapia no crescimento.

As alterações longitudinais no crescimento mandibular em 30 indivíduos com má oclusão de Classe II, $1^{\text {a }}$ divisão, não tratados ortodonticamente, foram comparadas com uma amostra de 35 indivíduos com oclusão normal (Iowa Longitudinal Facial Growth Study), desde a dentadura decídua até a dentadura permanente. A análise comparativa das telerradiografias obtidas anualmente demonstrou que as diferenças na posição e comprimento mandibular foram mais evidentes nos estágios precoces do desenvolvimento do que nos estágios tardios. Além disso, os resultados indicaram que o crescimento tendeu a ser quase similar entre os dois grupos, embora uma desarmonia entre as bases ósseas já esteja presente precocemente nas más oclusões de Classe II. Quando se analisou a diferença morfológica entre os dois grupos, verificou-se que durante o período de crescimento, os indivíduos portadores de má oclusão de Classe II apresentaram um perfil mais convexo, com mandíbulas mais retruídas, embora o grau de retrusão tenha diminuído ao longo do crescimento ${ }^{5}$.

O crescimento craniofacial nos indivíduos portadores de Classe II esquelética, não tratados ortodonticamente, também foi estudado por Chung e Wallace $^{11}$, que selecionaram 85 indivíduos (45 do gênero masculino e 40 do gênero feminino), pertencentes a dois centros de crescimento (Bolton-Brush e Burlington Growth Studies). Foram analisados longitudinalmente os cefalogramas dos 09 aos 18 anos, dividindo-se os pacientes em dois grupos distintos; quanto ao padrão de crescimento vertical (>360) e horizontal $(<270)$. Os resultados mostraram que houve uma redução progressiva da convexidade facial decorrente de uma rotação para frente da mandíbula, sendo o grau dessa redução intimamente relacionada com o padrão de 
crescimento, visto que esta foi mais evidente nos padrões horizontais. Nenhuma alteração no comportamento do crescimento foi observada quando comparados os gêneros masculino e feminino.

Destarte, o incremento ântero-posterior da mandíbula durante o surto de crescimento puberal é um fator determinante para correção de algumas desarmonias esqueléticas sagitais, contribuindo para o estabelecimento de um padrão facial mais equilibrado. No entanto, embora o incremento nas dimensões mandibulares se manifeste intensamente durante este período, existe uma grande variabilidade individual quanto à quantidade, velocidade e início deste surto de crescimento. O profissional deve assim utilizar-se de diversos artifícios para tentar predizer mais precisamente como ocorrerá este crescimento ${ }^{22}$.

Tal informação apresenta importância na área ortodôntica, uma vez que o diagnóstico e o plano de tratamento podem sofrer influência da estimativa da existência de um significativo crescimento

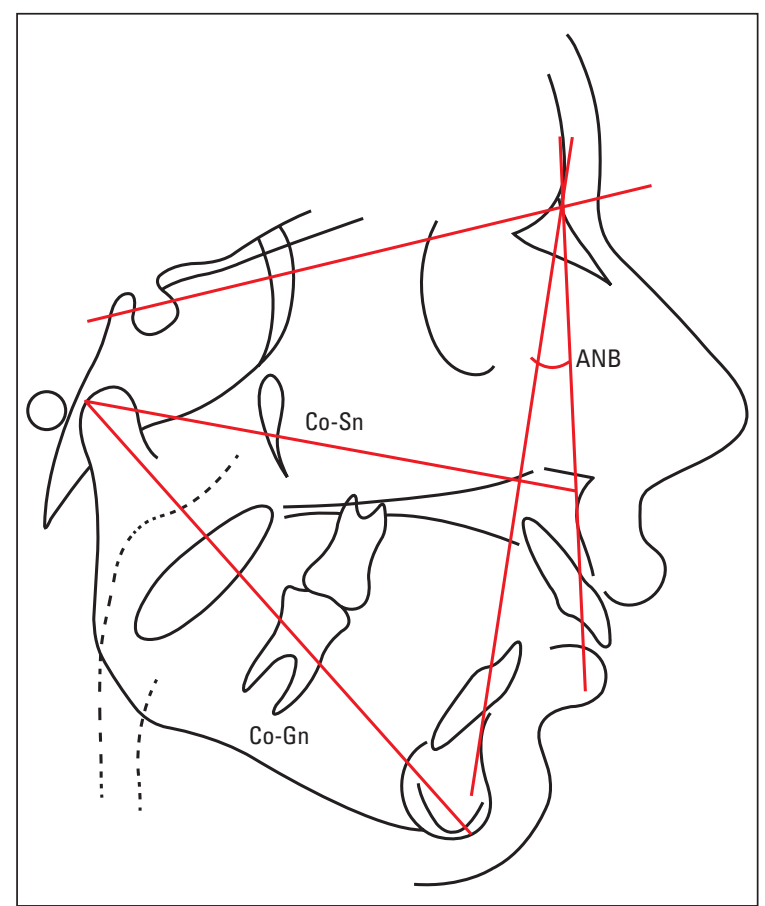

FIGURA 1 - Diagrama ilustrando as medidas utilizadas como critério para caracterizar a má oclusão de Classe II. craniofacial subseqüente. A escolha da época para a instituição de terapias que visem corrigir discrepâncias maxilo-mandibulares significativas deve levar em consideração o estágio de maturação do paciente, visto que este pode influenciar decisivamente no resultado do tratamento. Conseqüentemente, nos pacientes em crescimento, torna-se necessária a escolha do momento mais adequado para o início de uma abordagem terapêutica. Desta maneira, é possível reduzir consideravelmente o tempo de tratamento e assim torná-lo mais eficiente, por meio da determinação do surto de crescimento craniofacial do adolescente.

\section{PROPOSIÇÃO}

O presente estudo tem por objetivo avaliar a velocidade média de crescimento estatural e mandibular, bem como as suas correlações entre si, em indivíduos portadores de Classe II esquelética, não tratados ortodonticamente, acompanhados longitudinalmente dos 6 aos 16 anos de idade.

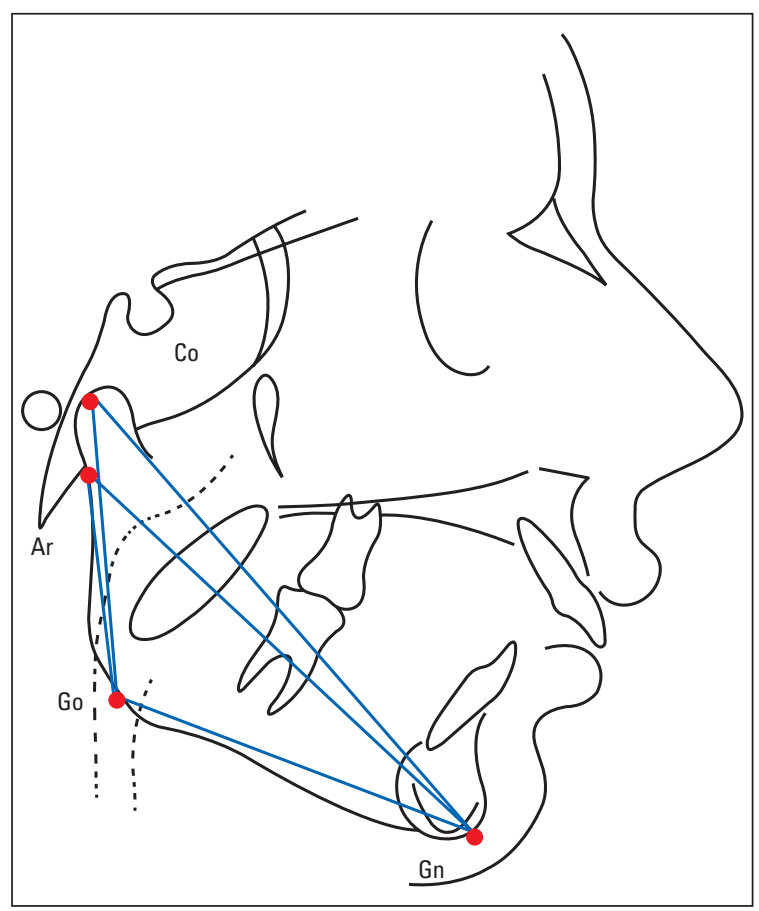

FIGURA 2 - Traçado cefalométrico ilustrando as grandezas lineares referentes ao comprimento mandibular total ( $\mathrm{Co}-\mathrm{Gn}$ e Ar-Gn), altura do ramo mandibular (Co-Go e Ar-Go) e comprimento do corpo da mandíbula (Go-Gn) utilizadas neste estudo para a avaliação do crescimento mandibular. 


\section{MATERIAL E MÉTODO}

Para realização do presente estudo, foram selecionados 30 indivíduos, sendo 17 do gênero masculino e 13 do gênero feminino, com Classe II esquelética, não tratados ortodonticamente, participantes do Burlington Growth Study, Departamento de Ortodontia da Faculdade de Odontologia, Universidade de Toronto, Canadá. Os indivíduos apresentam documentações seriadas longitudinais obtidas aos 6, 9, 12, 14 e 16 anos (idade cronológica), constando de:

a) Telerradiografia de perfil com os dentes em máxima intercuspidação habitual;

b) Ficha clínica com dados cadastrais e história médica e dentária.

A Classe II esquelética foi definida pelos seguintes critérios (Fig. 1):

1) ângulo ANB igual ou maior que $5^{\circ}$; e/ou

2) diferença entre o comprimento da mandíbula (Co-Gn) e o comprimento ântero-posterior do terço médio da face (Co-Sn) menor ou igual a $20 \mathrm{~mm}$.

Os traçados cefalométricos foram todos realizados por um mesmo operador, previamente calibrado. Após a sua confecção, procedeu-se a digitalização dos pontos cefalométricos (Quadro 1) com o auxílio de uma mesa digitalizadora, sendo as grandezas cefalométricas relativas ao crescimento

\footnotetext{
S Ponto central da sela túrcica

$\mathrm{N}$ Ponto mais anterior da sutura frontonasal

Sn Ponto localizado no contorno anterior maxilar, $2 \mathrm{~mm}$ abaixo do plano palatal

A Ponto mais profundo na concavidade do contorno anterior da maxila

B Ponto mais profundo na concavidade do contorno anterior da mandíbula

Co Ponto mais superior e posterior no contorno do côndilo mandibular

Ar Ponto situado na interseção do contorno posterior do côndilo mandibular com a base do osso occiptal

Gn Ponto determinado pela bissetriz resultante do encontro das linhas tangentes à borda inferior da mandíbula e ao ponto mais anterior do mento ósseo

Go Ponto obtido pela bissetriz resultante do encontro das linhas tangentes à borda inferior da mandíbula e ao ponto mais anterior do mento ósseo

QUADRO 1 - Pontos cefalométricos utilizados para a obtenção das medidas avaliadas no presente estudo.
}

mandibular mensuradas utilizando-se o software DentoFacial Planner Plus (DentoFacial Software Inc.-Toronto-Canadá). Para a verificação das alterações no crescimento mandibular, foram utilizadas grandezas lineares que envolveram o comprimento mandibular (Co-Gn e Ar-Gn), a altura do ramo da mandíbula (Co-Go e Ar-Go) e o comprimento do corpo mandibular (Go-Gn) (Quadro 2), (Fig. 2). Por meio de fichas clínicas, coletou-se os dados referentes à estatura dos pacientes nos diferentes intervalos de tempo.

Os dados relativos às alterações no comprimento mandibular, altura do ramo mandibular e comprimento do corpo mandibular, obtidos pelas grandezas lineares mensuradas nas telerradiografias em norma lateral, foram correlacionados às alterações na altura corporal registradas nas fichas clínicas com dados cadastrais dos pacientes nas idades de $6,9,12,14$ e 16 anos. No tratamento estatístico destes dados, utilizou-se o teste não-paramétrico de Friedman, ao nível de significância de 1\%, a fim de verificar a existência de diferenças entre as velocidades médias de incremento ao longo do período de avaliação, além de determinar o período de maior velocidade de crescimento para cada uma destas medidas analisadas. Foi também realizado a Análise de Correlação de Pearson ( $\mathrm{r})(\mathrm{p} \leq 0,05)$, no intuito de verificar a existência de correlação entre o

\footnotetext{
ANB Ângulo formado pela diferença entre os ângulos SNA e SNB, representando a relação sagital entre as bases ósseas

Co-Sn Distância linear entre o ponto Co e o ponto Sn, representando o comprimento ântero-posterior do terço médio da face

Co-Gn Distância linear entre o ponto Co e o ponto Gn, representando o comprimento mandibular

Ar-Gn Distância linear entre o ponto Ar e o ponto Gn, representando o comprimento mandibular

Co-Go Distância linear entre o ponto Co e o ponto Go, representando a altura do ramo mandibular

Ar-Go Distância linear entre o ponto Ar e o ponto Go, representando a altura do ramo mandibular

Go-Gn Distância linear entre o ponto Go e o ponto $\mathrm{Gn}$, representando o comprimento do corpo mandibular

QUADRO 2 - Grandezas cefalométricas angulares e lineares utilizadas para a seleção da amostra e para a avaliação do crescimento mandibular.
} 
crescimento estatural dos indivíduos e o crescimento mandibular (tanto para a amostra geral, como para cada gênero em particular).

Para determinação do cálculo do erro intraexaminador, 30 traçados cefalométricos de perfil foram aleatoriamente selecionados e novamente digitalizados e medidos através do programa
DentoFacial Planner Plus, com intervalo de uma semana entre a primeira e a segunda avaliação. $\mathrm{O}$ cálculo do erro foi determinado pelo Teste $t$ de Student para amostras pareadas, comparando-se os valores obtidos da primeira mensuração, com os valores encontrados na segunda mensuração, a um nível de significância de $1 \%$.

\begin{tabular}{|c|c|c|c|c|}
\hline \multirow{2}{*}{ Medida } & \multicolumn{4}{|c|}{ Velocidade Média de Incremento Anual (desvio-padrão) } \\
\hline & $6-9$ anos & $9-12$ anos & $12-14$ anos & $14-16$ anos \\
\hline Altura (cm/ano) & $5.65(0.61)^{A}$ & $5.21(1.05)^{\mathrm{B}}$ & $5.68(2.13)^{A}$ & $3.89(2.93)^{\mathrm{B}}$ \\
\hline Co-Gn (mm/ano) & $2.58(0.79)^{A}$ & $2.41(0.77)^{A}$ & $2.78(1.70)^{\mathrm{A}}$ & $2.38(2.01)^{A}$ \\
\hline Ar-Gn (mm/ano) & $2.35(0.65)^{\mathrm{AB}}$ & $2.02(0.60)^{\mathrm{B}}$ & $2.62(1.16)^{\mathrm{A}}$ & $2.07(1.65)^{A B}$ \\
\hline Co-Go (mm/ano) & $1.14(0.74)^{\mathrm{B}}$ & $1.05(0.73)^{\mathrm{B}}$ & $1.56(1.17)^{\mathrm{AB}}$ & $2.13(1.40)^{\mathrm{A}}$ \\
\hline Ar-Go (mm/ano) & $0.70(0.58)^{\mathrm{B}}$ & $0.71(0.74)^{\mathrm{B}}$ & $1.23(1.04)^{\mathrm{A}}$ & $1.67(1.20)^{\mathrm{A}}$ \\
\hline Go-Gn (mm/ano) & $2.20(0.93)^{\mathrm{A}}$ & $1.61(0.72)^{\mathrm{B}}$ & $1.67(1.21)^{\mathrm{B}}$ & $1.40(1.28)^{\mathrm{B}}$ \\
\hline
\end{tabular}

* Médias seguidas de mesma letra não diferem entre si.

Tabela 2 - Médias, desvios-padrão e Análise de Variância (teste não-paramétrico de Friedman) da velocidade média de incremento anual das dimensões avaliadas para os diferentes intervalos de tempo, nos indivíduos do gênero feminino ( $n=13)$.

\begin{tabular}{|c|c|c|c|c|}
\hline \multirow{2}{*}{ Medida } & \multicolumn{4}{|c|}{ Velocidade Média de Incremento Anual (desvio-padrão) } \\
\hline & $6-9$ anos & $9-12$ anos & $12-14$ anos & $14-16$ anos \\
\hline Altura $(\mathrm{cm})$ & $5.57(0.55)^{\mathrm{A}}$ & $5.80(1.18)^{\mathrm{A}}$ & $4.44(1.73)^{\mathrm{B}}$ & $1.49(1.29)^{\mathrm{c}}$ \\
\hline Co-Gn (mm) & $2.71(0.88)^{\mathrm{A}}$ & $2.79(0.77)^{\mathrm{A}}$ & $2.51(1.71)^{\mathrm{AB}}$ & $1.45(1.02)^{\mathrm{B}}$ \\
\hline $\operatorname{Ar}-\mathrm{Gn}(\mathrm{mm})$ & $2.29(0.38)^{\mathrm{A}}$ & $2.34(0.67)^{\mathrm{A}}$ & $2.45(0.94)^{\mathrm{A}}$ & $0.86(0.76)^{\mathrm{B}}$ \\
\hline Co-Go (mm) & $1.53(0.82)^{\mathrm{A}}$ & $1.44(0.80)^{\mathrm{A}}$ & $1.00(0.85)^{\mathrm{A}}$ & $1.81(0.97)^{\mathrm{A}}$ \\
\hline $\operatorname{Ar}-\mathrm{Go}(\mathrm{mm})$ & $0.92(0.68)^{A}$ & $1.15(0.83)^{A}$ & $0.92(0.62)^{A}$ & $1.05(0.88)^{\mathrm{A}}$ \\
\hline Go-Gn (mm) & $1.63(0.67)^{\mathrm{A}}$ & $1.79(0.74)^{\mathrm{A}}$ & $1.75(1.29)^{\mathrm{A}}$ & $0.48(0.72)^{\mathrm{B}}$ \\
\hline
\end{tabular}

* Médias seguidas de mesma letra não diferem entre si.

Tabela 3 - Médias, desvios-padrão e Análise de Variância (teste não-paramétrico de Friedman) da velocidade média de incremento anual das dimensões avaliadas para os diferentes intervalos de tempo, nos indivíduos do gênero masculino (n=17).

\begin{tabular}{|c|c|c|c|c|}
\hline \multirow{2}{*}{ Medida } & \multicolumn{4}{|c|}{ Velocidade Média de Incremento Anual (desvio-padrão) } \\
\hline & $6-9$ anos & $9-12$ anos & $12-14$ anos & $14-16$ anos \\
\hline Altura $(\mathrm{cm})$ & $5.79(0.66)^{\mathrm{A}}$ & $4.76(0.68)^{\mathrm{B}}$ & $6.63(1.95)^{\mathrm{A}}$ & $5.58(2.55)^{A B}$ \\
\hline Co-Gn (mm) & $2.49(0.73)^{A}$ & $2.12(0.64)^{\mathrm{A}}$ & $3.10(2.30)^{A}$ & $2.98(1.71)^{\mathrm{A}}$ \\
\hline $\operatorname{Ar}-\mathrm{Gn}(\mathrm{mm})$ & $2.40(0.81)^{\mathrm{A}}$ & $1.77(0.40)^{\mathrm{B}}$ & $3.00(1.55)^{\mathrm{A}}$ & $2.75(1.31)^{\mathrm{A}}$ \\
\hline Co-Go (mm) & $0.84(0.52)^{B}$ & $0.74(0.50)^{\mathrm{B}}$ & $2.37(1.64)^{\mathrm{A}}$ & $1.99(1.22)^{\mathrm{A}}$ \\
\hline $\operatorname{Ar}-G_{0}(\mathrm{~mm})$ & $0.53(0.43)^{\mathrm{B}}$ & $0.45(0.50)^{\mathrm{B}}$ & $2.07(1.31)^{\mathrm{A}}$ & $1.47(1.24)^{\mathrm{A}}$ \\
\hline Go-Gn (mm) & $2.51(0.95)^{\mathrm{A}}$ & $1.59(0.78)^{\mathrm{B}}$ & $2.60(1.17)^{\mathrm{A}}$ & $1.60(1.18)^{\mathrm{B}}$ \\
\hline
\end{tabular}

* Médias seguidas de mesma letra não diferem entre si. 


\begin{tabular}{|c|c|c|c|}
\hline Medida & r Feminino (p) & r Masculino (p) & r Total (p) \\
\hline \multicolumn{4}{|l|}{$6-9$ anos } \\
\hline Co-Gn & $0,094(0,75)$ & $0,209(0,42)$ & $0,119(0,53)$ \\
\hline Ar-Gn & $0,096(0,76)$ & $0,269(0,52)$ & $0,170(0,37)$ \\
\hline Co-Go & $0,053(0,86)$ & $0,156(0,55)$ & $0,167(0,38)$ \\
\hline Ar-Go & $0,113(0,71)$ & $0,398(0,11)$ & $0,283(0,13)$ \\
\hline Go-Gn & $0,160(0,60)$ & $0,055(0,83)$ & $0,116(0,54)$ \\
\hline \multicolumn{4}{|l|}{9 - 12 anos } \\
\hline Co-Gn & $0,333(0,27)$ & $0,054(0,84)$ & $0,390\left(0,03^{*}\right)$ \\
\hline Ar-Gn & $0,475(0,10)$ & $0,320(0,21)$ & $0,563\left(0,01^{*}\right)$ \\
\hline Co-Go & $0,170(0,58)$ & $0,021(0,94)$ & $0,282(0,13)$ \\
\hline Ar-Go & $0,188(0,72)$ & $0,176(0,50)$ & $0,305(0,10)$ \\
\hline Go-Gn & $0,070(0,82)$ & $0,217(0,40)$ & $0,123(0,52)$ \\
\hline \multicolumn{4}{|l|}{12 - 14 anos } \\
\hline Co-Gn & $0,176(0,56)$ & $0,687(0,01 *)$ & $0,539\left(0,01^{*}\right)$ \\
\hline Ar-Gn & $0,486(0,09)$ & $0,758\left(0,01^{*}\right)$ & $0,634(0,01 *)$ \\
\hline Co-Go & $0,165(0,59)$ & $0,187 \quad(0,47)$ & $0,279(0,14)$ \\
\hline Ar-Go & $0,266(0,38)$ & $0,615(0,01 *)$ & $0,560\left(0,01^{*}\right)$ \\
\hline Go-Gn & $0,481(0,09)$ & $0,537\left(0,03^{*}\right)$ & $0,404\left(0,03^{*}\right)$ \\
\hline \multicolumn{4}{|l|}{$14-16$ anos } \\
\hline Co-Gn & $0,492(0,10)$ & $0,573\left(0,02^{*}\right)$ & $0,657\left(0,01^{*}\right)$ \\
\hline Ar-Gn & $0,553(0,06)$ & $0,596(0,01 *)$ & $0,774\left(0,01^{*}\right)$ \\
\hline Co-Go & $0,372(0,23)$ & $0,311(0,22)$ & $0,370\left(0,04^{*}\right)$ \\
\hline Ar-Go & $0,440(0,65)$ & $0,580(0,01 *)$ & $0,594\left(0,01^{*}\right)$ \\
\hline Go-Gn & $0,411(0,16)$ & $0,075(0,78)$ & $0,466\left(0,01^{*}\right)$ \\
\hline
\end{tabular}

* Correlação significativa.

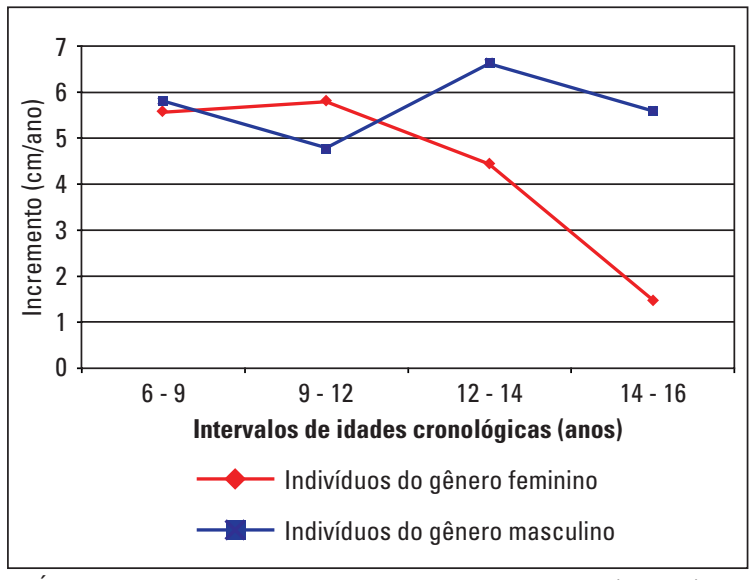

GRÁFICO 1 - Velocidade média de crescimento estatural anual (cm/ano) para os indivíduos do gênero feminino e masculino, de acordo com os intervalos de tempo estudados.

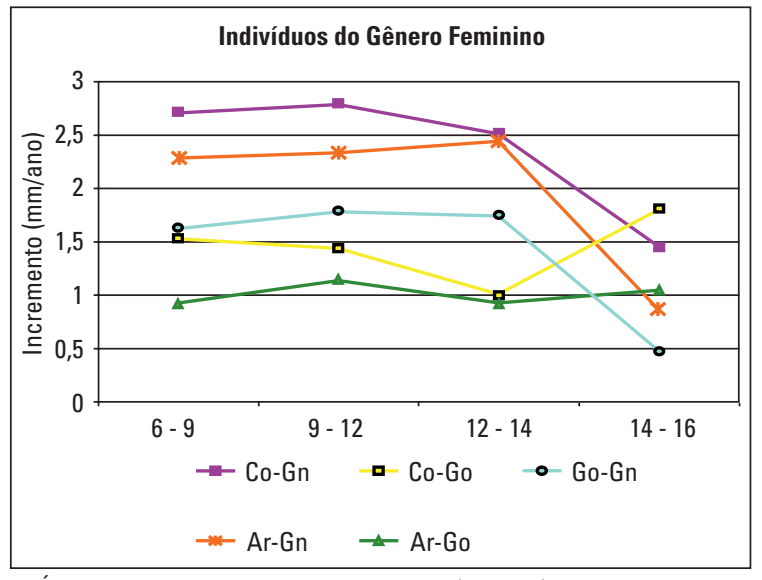

GRÁFICO 2 - Velocidade média de incremento (mm/ano) das dimensões mandibulares para os indivíduos do gênero feminino, de acordo com os intervalos de tempo estudados. 


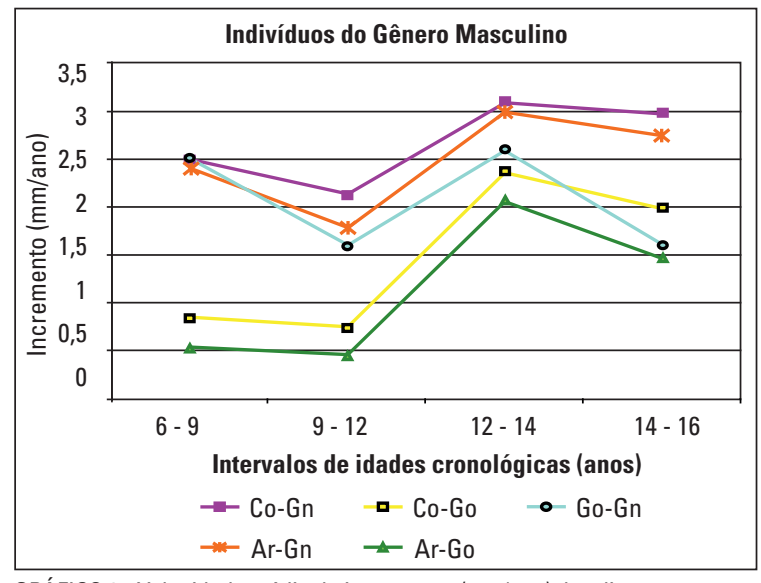

GRÁFICO 3 - Velocidade média de incremento (mm/ano) das dimensões mandibulares para os indivíduos do gênero masculino, de acordo com os intervalos de tempo estudados.

\section{RESULTADOS}

Através do cálculo do erro metodológico (avaliação do erro intra-investigador), foi verificado que o erro combinado não excedeu $0,4 \mathrm{~mm}$ de comprimento para todas as variáveis investigadas $(\mathrm{p} \leq 0,01)$.

Através dos resultados do teste não-paramétrico de Friedman, verificou-se que apenas a medida Co-Gn não apresentou diferença estatística significativa quanto à sua taxa de incremento anual nos diferentes intervalos de tempo. O restante das medidas teve seus valores diferentes entre as idades $(\mathrm{p} \leq 0,01)$ (Tab. 1).

Através dos resultados do teste não-paramétrico de Friedman, verificou-se que apenas as medidas Co-Go e Ar-Go não apresentaram diferença estatística quanto a sua taxa de incremento anual nos diferentes intervalos de tempo. O restante das medidas teve seus valores diferentes entre as idades $(\mathrm{p} \leq 0,01)$ (Tab. 2).

Através dos resultados do teste não-paramétrico de Friedman, verificou-se que apenas a medida CoGn não apresentou diferença estatística quanto a sua taxa de incremento anual nos diferentes intervalos de tempo. $\mathrm{O}$ restante das medidas teve seus valores diferentes entre as idades ( $\mathrm{p} \leq 0,01)$ (Tab. 3).

Para todas as correlações significativas, obser- va-se uma relação direta entre as velocidades de crescimento em altura e as velocidades de incremento das dimensões mandibulares, ou seja, quanto maior a velocidade de crescimento em altura maior tende a ser a velocidade de incremento das dimensões mandibulares. (Tab. 4).

\section{DISCUSSÃO}

Uma das estratégias do tratamento ortodôntico durante a adolescência, em casos onde existem discrepâncias esqueléticas, seria a utilização racional das mudanças ocorridas no crescimento facial do paciente. Através de um manejo eficiente do complexo craniofacial em crescimento, os resultados podem ser obtidos de maneira otimizada e com um período de tratamento relativamente mais curto ${ }^{4}$.

Os ortodontistas comumente estão interessados em definir as mudanças nos diversos componentes da estrutura craniofacial. Atenção principal é dada às mudanças ocorridas na mandíbula, uma vez que, dentre os componentes morfológicos da face, o osso mandibular é, em termos relativos, a estrutura que apresenta o crescimento mais significativo durante toda a adolescência. Uma série de estudos demonstrou a existência de um surto de crescimento mandibular durante o período pubera ${ }^{17,18,20,23}$. Através destes achados, verificou-se que este surto de crescimento das dimensões mandibulares seria expresso como uma mudança na posição espacial da mandíbula para frente e para baixo $0^{4,20}$.

Vários pesquisadores procuraram determinar o momento, a duração, a magnitude e a direção de crescimento mandibular, relacionando-os a outras estruturas corpóreas, como por exemplo, a altura corporal 1,2,3,4,10,18,26,27,35,36. Segundo esses autores, a altura corporal do indivíduo, um dos fatores determinantes da sua idade morfológica, constituiu um bom indicador de maturidade, podendo assim ser adequadamente utilizada desde a tenra infância até o início da idade adulta. Tal avaliação do nível de desenvolvimento biológico constitui um método simples e de fácil aferição ${ }^{6}$.

A determinação da existência do surto de 
crescimento corporal é de suma importância, visto que, como regra geral, o crescimento do complexo craniofacial também ocorrerá nesta época. Um dos exemplos mais evidentes desta possibilidade é a questão de decidir quando tratar a má oclusão de Classe II. Esse tópico é ainda bastante controverso na literatura ortodôntica. Alguns autores sugerem que o tratamento deve iniciar-se na dentadura mista precoce $e^{37,39,40}$, enquanto outros preferem aguardar até a irrupção de toda a dentadura permanente ${ }^{19,25}$.

Muitos estudos já foram realizados avaliando a relação existente entre as dimensões mandibulares e a altura corporal dos indivíduos. No entanto, poucas pesquisas procuraram avaliar a existência de tal relação em indivíduos portadores de Classe II esquelética. Deste modo, torna-se primordial a realização de tais investigações, uma vez que, nestes pacientes, o crescimento da mandíbula ocorre de maneira diferenciada dos indivíduos com padrões normais de crescimento (Classe I). Além disso, o tratamento ortodôntico/ortopédico desses pacientes implica no aproveitamento máximo do potencial de crescimento mandibular, necessitando assim a compreensão plena de todas as características inerentes ao desenvolvimento do complexo craniofacial.

Desta forma, os resultados aqui apresentados oferecem importantes implicações no que tange o conhecimento sobre a magnitude e a taxa de incremento médio das medidas mandibulares em indivíduos com Classe II esquelética, bem como a relação existente entre o crescimento mandibular e o crescimento em altura destes indivíduos.

Pode ser observado através deste estudo que a altura e as dimensões mandibulares apresentam acréscimos durante todo o período de crescimento (Fig. 3). A maior velocidade média de incremento anual das dimensões que representam o comprimento mandibular (Co-Gn e Ar-Gn) tende a ocorrer no mesmo período que o máximo incremento em altura para os indivíduos de ambos os gêneros (Tab. 1). Este resultado é consistente com outros achados da literatura ${ }^{18,35}$, que encon-

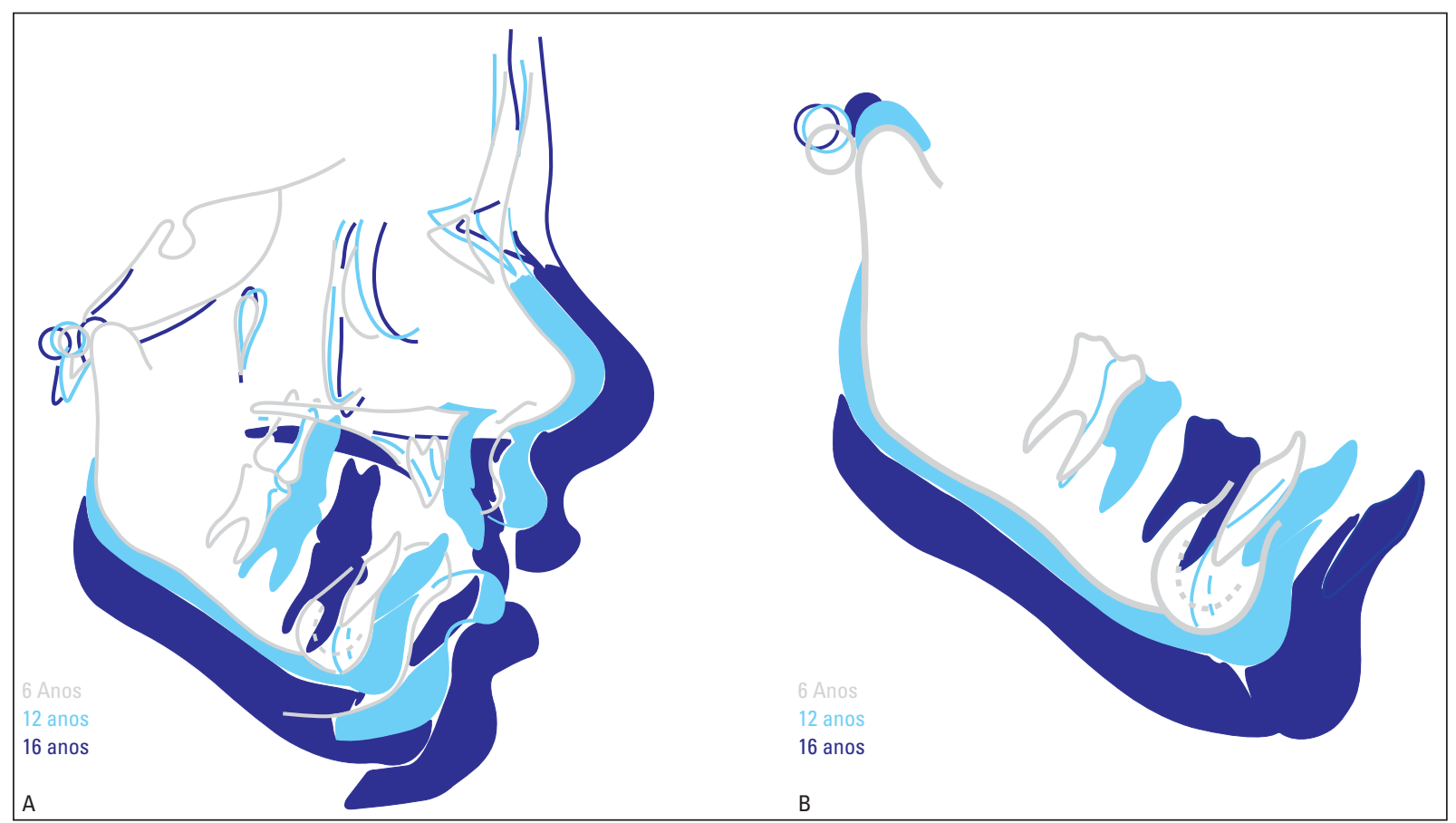

FIGURA 3 - Sobreposição cefalométrica total. A) (linha S-N com registro em S) e parcial de mandíbula. B) (sobre o ponto articular - Ar) entre os traçados aos 6 anos, 12 anos e 16 anos do indivíduo J.C., do gênero masculino. 
traram relação entre o pico de crescimento destas medidas mandibulares e o pico de crescimento em estatura. Já o pico de crescimento do ramo mandibular (avaliado através das medidas Co-Go e Ar-Go) ocorreu após o máximo incremento em altura destes indivíduos, e o pico de crescimento do corpo mandibular (avaliado através da medida Go-Gn) antes deste pico de crescimento estatural. Isto pode ser explicado pelo fato que o crescimento vertical da mandíbula ocorre mais tardiamente e continua por mais tempo que seu o crescimento ântero-posterior, em ambos os gêneros ${ }^{28}$.

O pico de crescimento estatural para o gênero feminino coincidiu com o maior incremento médio anual do comprimento mandibular (Co-Gn), da altura do ramo mandibular (Ar-Go) e do comprimento do corpo mandibular (Go-Gn), ocorrendo no período entre 9 e 12 anos de idade cronológica, conforme pode ser observado através da comparação entre os gráficos 1 e 3 e na análise da tabela 2. O comprimento mandibular mensurado através da medida Ar-Gn e a altura do ramo mandibular mensurada pela medida Co-Go mostraram comportamento diferentes, indicando picos de crescimento mais tardios, entre os 12 e 14 anos e entre os 14 e 16 anos, respectivamente.

Já para o gênero masculino, o pico de crescimento estatural, bem como o maior incremento médio anual de todas as dimensões mandibulares avaliadas (Co-Gn, Ar-Gn, Co-Go, Ar-Go e Go-Gn) ocorreu no período entre 12 e 14 anos de idade cronológica, conforme observado através da comparação entre os gráficos 2 e 4 e na tabela 3.

Embora o comprimento mandibular total, a altura do ramo mandibular e o comprimento do corpo mandibular compreendam dimensões de uma mesma estrutura, a correlação entre as suas velocidades de crescimento e a velocidade de crescimento estatural apresenta algumas diferenças. Isto acontece em decorrência da forma geométrica da mandíbula, onde o seu comprimento total é resultante de três fatores básicos: o comprimento do ramo, o ângulo goníaco e o comprimento do corpo mandibular ${ }^{15}$.
Destarte, em termos gerais, o incremento do comprimento mandibular total (avaliado pelas medidas Co-Gn e Ar-Gn) tendeu a apresentar uma correlação mais alta e consistente com o incremento em altura dos indivíduos avaliados (Tab. 4). Observou-se também que, para os indivíduos do gênero feminino, a correlação entre a velocidade de crescimento estatural e a velocidade de crescimento das dimensões mandibulares não foi significativa em todos os intervalos de tempo avaliados. Para os indivíduos do gênero masculino, esta correlação existiu e foi significativa para a maioria das dimensões mandibulares avaliadas, principalmente no período onde ocorreu o pico de crescimento tanto estatural como mandibular (entre os 12 e os 14 anos). As medidas que apresentaram maior correlação entre a sua velocidade média de incremento anual e a velocidade média de incremento estatural foram as que representam o comprimento mandibular (Ar-Gn, r=0,758 / Co-Gn, r=0,687).

Pôde ainda ser constatado que as dimensões mandibulares determinadas a partir do ponto Articular apresentaram um maior índice de correlação com o crescimento estatural quando comparada às dimensões avaliadas pelo ponto Condílio. Esta diferença pode ser atribuída pela dificuldade na localização do ponto Condílio e à maior acuracidade nas medidas que utilizaram o ponto Articular.

Entretanto, o objetivo principal desta análise de correlação entre o incremento estatural e o incremento destas diversas medidas consiste na possibilidade da predição do crescimento mandibular. Horowitz e Hixon ${ }^{17}$ relataram que coeficientes de correlação menores do que 0,7 apresentam pouco valor predictivo quando aplicados para a população como um todo. Isto porque, quando avaliado o coeficiente de determinação $\left(R=r^{2}\right)$ entre estes fatores, observa-se que menos de metade da dimensão de uma destas medidas pode ser considerada como determinada diretamente pela dimensão da outra. Desta forma, mesmo havendo correlações significativas entre a taxa de crescimento em 
altura e a taxa de crescimento de certas medidas mandibulares para os indivíduos analisados, estes dados devem ser interpretados com cautela.

Através da análise da tabela 4, observa-se que somente o crescimento anual da dimensão Articular-Gnátio apresentou um coeficiente de correlação maior que 0,7 , quando comparado com o crescimento anual em estatura dos indivíduos do gênero masculino, no intervalo de tempo entre os 12 e os 14 anos de idade $(\mathrm{r}=0,758)$. Para os indivíduos do gênero feminino, esta predição da taxa de crescimento mandibular fica ainda mais restrita, uma vez que as correlações realizadas, embora se apresentem de maneira direta (ou seja, uma maior velocidade média de crescimento estatural é acompanhada de uma maior velocidade média de incremento das medidas mandibulares), não foram significativas do ponto de vista estatístico $(\mathrm{p}>0,05)$.

Sendo assim, verifica-se que a determinação do pico de crescimento em altura de um indivíduo, o qual pode ser facilmente constatado sem a necessidade de exames complementares, constitui um dado adicional quando da tentativa de predição do pico de crescimento das medidas mandibulares avaliadas, porém não totalmente preciso. Bishara et al. ${ }^{4}$ recomendam que outros métodos devem ser empregados para uma avaliação mais criteriosa do padrão de crescimento mandibular do paciente. Além da correlação com o crescimento estatural, o incremento das dimensões mandibulares deve ser analisado e previsto de maneira mais precisa e confiável por meio de uma avaliação da idade esquelética do paciente (através de radiografias carpais), pela sobreposi- ções de imagens cefalométricas obtidas em intervalos de tempo pré-determinados e/ou ainda, mais raramente utilizado, o método bioquímico para avaliar o nível de hormônio de crescimento circulante na corrente sanguínea.

\section{CONCLUSÕES}

- O pico de crescimento estatural, bem como o maior incremento médio anual da maioria das dimensões mandibulares avaliadas (Co-Gn, Ar-Go e Go-Gn), ocorreu, para o gênero feminino, no período entre 9 e 12 anos de idade cronológica.

- Para o gênero masculino, o pico de crescimento estatural, bem como o maior incremento médio anual de todas as dimensões mandibulares avaliadas (Co-Gn, Ar-Gn, Co-Go, Ar-Go e Go$\mathrm{Gn})$ ocorreu no período entre 12 e 14 anos de idade cronológica.

- A velocidade de incremento no comprimento da mandíbula (Ar-Gn e Co-Gn), dentre todas as medidas mandibulares avaliadas, demonstrou a correlação mais consistente com a velocidade de crescimento em altura destes indivíduos, principalmente para aqueles do gênero masculino.

\section{AGRADECIMENTOS}

"Este estudo tornou-se possivel pelo uso de material do Burlington Growth Centre (Centro de Crescimento Burlington) Faculdade de Odontologia, Universidade de Toronto, que foi suportado por fundos fornecidos por Concessão (1) ( N 605-7-299) Concessão de Saúde Nacional (Canadá), (recoleta de dados): (2) Província de Concessão de Ontário PR 33 e (3) Fundo Varsity (para habitação e recoleta)". 


\title{
Longitudinal study of the relationship between mandibular growth and height growth in subjects with skeletal Class II
}

\begin{abstract}
The height growth of children and teenagers, as well as its relationship with the rate of craniofacial growth, are of large interest to the orthodontist, since the indication of a therapy to control jaw development is based on the study of the skeletal age of the patient and subsequent somatic maturation. Therefore, this study aimed at evaluating the correlation between height growth and mandibular growth of 30 subjects presenting with skeletal Class II, with no orthodontic treatment, which were longitudinally followed-up through the Burlington Growth Study of Canada. The serial records were obtained at 6, 9, 12, 14 and 16 years of chronological age and comprised lateral cephalograms, clinical records and personal information. The results indicated that the height growth spurt, as well as the largest mean increase in the mandibular measurements, occurred from 9 to 12 years of age for the females and from 12 to 14 years of age for the males. Moreover, it was also observed that, among all study measurements, the rate of increase of the mandibular length demonstrated the strongest correlation with the mean rate of height growth of these individuals, specially for the males during their growth spurt $(r=0,758)$.
\end{abstract}

Key words: Class II. Mandibular growth. Height growth. Facial growth spurt.

\section{REFERÊNCIAS}

1. ANDERSON,D.L.; THOMPSON, G.W.; POPOVICH, F. Adolescent variation in weight, height, and mandibular length in 111 females. Human Biology, London, v. 47, no. 3, p. 279-285, Sept.1975.

2. ANDERSON, D. L.; THOMPSON, G. W.; POPOVICH, F. Interrelation of dental maturity, skeletal maturity, height and weight from age 4 to 14 years. Growth, Philadelphia, v. 39, no. 4, p. 453-462, Nov. 1975.

3. BAMBHA, J. K. Longitudinal cephalometric roentgenographic study of face and cranium in relation to body height. J Am Dent Assoc, Chicago, v. 63, p. 776-779, Dec. 1961.

4. BISHARA, S. E. et al. Longitudinal changes in standing height and mandibular parameters between the ages of 8 and 17 years. Am J Orthod, St. Louis, v. 80, no. 2, p. 115-135, 1981.

5. BISHARA, S.E. Mandibular changes in persons with untreated and treated Class II Division 1 malocclusion. Am J Orthod Dentofacial Orthop, St. Louis, v. 113, no. 6, p. 661-673, June 1998.

6. BISHARA, S. E. Textbook in Orthodontics. Philadelphia: W. B. Saunders Company, 2001.

7. BJÖRK, A. Facial growth in man, studied with the aid of metallic implants. Acta Odontol Scand, Oslo, v.13, no. 1, p. 9-34, 1955.

8. BJÖRK, A. Variations in the growth pattern of the human mandibule: longitudinal radiographic study by the implant method. J Dent Res, Alexandria, v. 42, p. 400-411, 1963. Supll. 1.

9. BJÖRK, A. Timing of interceptive orthodontic measures based on stages of maturation. Trans Eur Orthod Soc, London, v.48, no. 2, p. 61-74, 1972.

10. BJÖRK, A.; HELM, S. Prediction of age of maximum pubertal growth in body heigth. Angle Orthod, Appleton, v. 37, no. 2, p. 134-143, 1967.
11. CHUNG, C. H.; WONG, W. W. Craniofacial growth in untreated skeletal Class II subjects: a longitudinal study. Am J Orthod Dentofacial Orthop, St. Louis, v. 122, no. 6, p. 619-626, Dec. 1998.

12. DAIGLE, D. J. A study of the timing and relationship of the pubertal growth acceleration between the maxila and stature. Thesis (Diploma of Orthodontics) - Faculty of Dentistry, University of Toronto, Toronto, 1974.

13. DALE, J. Interceptive guidance of occlusion with enphasis on diagnosis. In: GRABER, T. M.; VANARSDALL, R. L. Orthodontics, current principles and techiniques. 2nd ed. St. Louis: C. V. Mosby, 1994.

14. GRAVE, K. C.; BROWN, T. Skeletal ossification and the adolescent growth spurt. Am J Orthod, St. Louis, v. 69, no. 6, p. 611619, Dec. 1976.

15. GRIWALSKY, A. A study of the correlation of maturity status as determined by body stature and by mandibular linear dimensions from mesurements of canadian females during childhood and adolescence. Thesis (Diploma of Orthodontics) - Faculty of Dentistry, University of Toronto, Toronto, 1966.

16. HIOKI, M. A longitudinal study of the relationship between general body growth and facial growth. J Japan Orthod Soc, Tokyo, v. 25, no. 1, p. 1-30, Jan. 1966.

17. HOROWITZ, S. L.; HIXON, E.H. The nature of orthodontic diagnosis. St. Louis: C. V. Mosby, 1966.

18. HUNTER, C. J. The correlation of facial growth with body height and skeletal maturation at adolescence. Angle Orthod, Appleton, v. 36, no.1, p. 44-54, 1966.

19. KOPECKY, G. R.; FISHMAN, L. S. Timing of cervical headgear treatment based on skeletal maturation. Am J Orthod Dentofacial Orthop, St. Louis, v. 104, no. 2, p. 162-169, Feb. 1993. 
20. LIMA, E. M. S. Avaliação do crescimento dos ossos maxilares e da mandíbula em indivíduos portadores de Classe II esquelética. 1999. Tese (Doutorado) - Faculdade de Odontologia, Universidade Federal do Rio de Janeiro, Rio de Janeiro, 1999.

21. McNAMARA Jr., J. A. Components of Class II maloclusiuon in children 8-10 years of age. Angle Orthod, Appleton, v. 51, no. 3, p. 177-202, 1981

22. MITANI, H.; SATO, K. Comparision of mandibular growth with other variables during puberty. Angle Orthod, Appleton, v. 62, no. 3, p. 217-222, Mar. 1992

23. MITANI, H. Prepubertal growth of mandibular prognathism. Am J Orthod, St. Louis, v. 80, no. 5, p. 546-553, Nov. 1981.

24. NANDA, R. S. The rates of growth of several facial components mesured from serial cephalometric roetnograms. Am J Orthod, St. Louis, v. 41, no. 6, p. 658-673, Dec. 1955.

25. PANCHERZ, H.; HAAG, V. Dentofacial orthopedics in relation to somatic maturation. An analysis of 70 consecutive cases with the Herbst appliance. Am J Orthod, St. Louis, v. 88, no. 4, p. 273287, Oct. 1985.

26. PIKE, J. B. A serial investigation of the facial and statural growth in 7 to 12 years old children. Angle Orthod, Appleton, v. 38, no. 2, p. 63-73, 1973

27. PILESKI, R. C. A.; WOODSIDE, D. G.; JAMES, G. A. Relatioship of the ulnar sesamoid bone and maximum mandibular growth velocity. Angle Orthod, Appleton, v. 43, no. 2, p.162-170, Apr. 1973.

28. PROFFIT, W. R. Ortodontia contemporânea. 3. ed. Rio de Janeiro: Ganabara Koogan. 2002.

29. REED, R.R. A longitudinal growth study relating mandibular length and skeletal maturation in children 9 to 16 years of age. 1968. Thesis (Master of Science in Orthodontics)-Faculty of Dentistry, State University of New York, New York, 1968.

30. SATO, K.; MITO, T.; MITANI, H. An accurate method of predicting mandibular growth potential based on bone maturity. Am J Orthod Dentofacial Orthop, St. Louis, v. 120, no. 3, p. 286290, Sept. 2001
31. SEIDE, L. J. The relationship of dentofacial grath and skeletal maturation to malocclusion. Am J Orthod, St. Louis, v. 45 no. 11, p. 801-816, Nov. 1959

32. SINGH, I. J.: SAVARA, B. S.; NEWMAN, M.T. Growth in the skeletal and non-skeletal components of head width from 9 to 14 years of age. Human Biol, London, v. 39, p. 182-191, 1967.

33. SIQUEIRA, V. C. V. et al. O emprego das radiografias da mão e do punho no diagnóstico ortodôntico. R Dental Press Ortodon Ortop Facial, Maringá, v. 4, n. 3, p. 20-29, maio/jun. 1999.

34. TANNER, J. M. Growth at adolecence. 2nd ed. Oxford: Blackwell, 1966

35. THOMPSON, G. W. ; POPOVICH, F. Relatioship of mandibular measurements to stature and weight in humans. Growth, [S. I.], v. 38, p.187-196, 1974

36. THOMPSON, G. W.; POPOVICH, F.; ANDERSON, D. L. Maximum growth changes in mandibular length, stature and weight. Hum Biol, London, v. 48, no. 2, p. 285-293, 1976

37. TULLOCH, J. F.C; PHILLIPS, C.; PROFFIT, W. R. Benefit of early Class II treatment: progress repot of a two phase randomized clinical trial. Am J Orthod Dentofacial Orthop, St. Louis, v. 113, no. 1, p. 62-72, Jan. 1998.

38. URSI, W. J. S. Determinação da maturidade esquelética através de radiografias carpais: sua importância no tratamento ortodôntico-ortopédico. In: INTERLANDI, S. Ortodontia: bases para a iniciação. 4. ed. São Paulo: Artes Médicas, 1999. cap.19, p. 377 -392 .

39. WIESLANDER, L. Intensive treatment of severe Class II malocclusion with a headgear-Herbst aplliance in the early mixed dentition. Am J Orthod, St. Louis, v. 104, no. 4, p. 319-329, Oct. 1993.

40. WIESLANDER, L. Early or late cervical tractin therapy of Class II maloclusion in the mixed dentition. Am J Orthod, St. Louis, v. 67, no. 4, p. 432-439, Apr. 1975

\section{Endereço para correspondência}

Guilherme Thiesen

Clínica Odontológica Santa Mônica

Av. Madre Benvenuta, n 1285 - Bairro Santa Mônica

CEP: 88035 - 000

Florianópolis - SC

guithiesen@hotmail.com 